\title{
(5) Anti-Acetylcholine Receptor Antibodies and Myasthenia Gravis.
}

\author{
Hiroshi Nishitani, MD \\ Department of Neurology, Utano National Hospital, Kyoto, Japan
}

The author reported the relationship between the titers of antiacetylcholine receptor (AChR) antibodies and clinical state in a series of myasthenic patients using two theoretically different assay systems in order to elucidate their correspondence and discrepancy with clinical conditons.

One hundred and forty-one myasthenic patients out of 210 , namely $65 \%$ showed positive result by immunoprecipitation method, assuming that the antibody recognizes antigenic determinants other than toxin binding sites in AChR protein, thus constitutes of 'non-inhibitory' antibody.

We adopt a new classification where the patients are classified into familial and non-familial types, the latter being devided into thymoma and non-thymoma groups. In the non-thymoma group the patients are classified into four types; ocular, generalized mild, moderate and severe types (Fig. 1).

Serial changes of the titers of anti-AChR antibody assayed by immunoprecipitation method showed close and parallel fluctuation associated with clinical changes; namely yearly decrease after complete thymectomy, monthly decrease during steroid therapy, weekly decrease in neonatal myasthenia and adrupt changes during. myasthenic crisis.

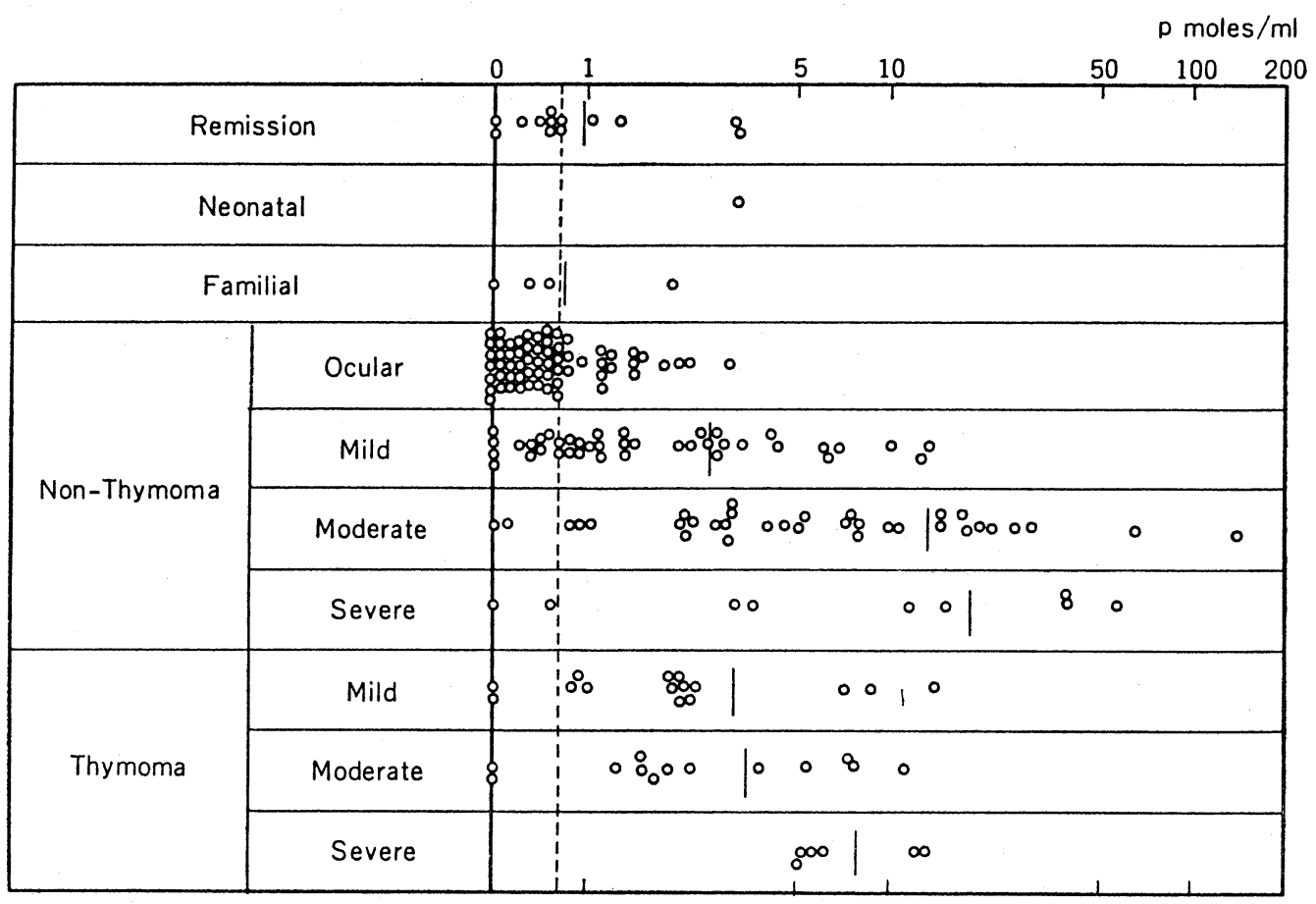

Fig. 1. Relationship between non-inhibitory activity using immunoprecipitation assay in myasthenic patients and their clinical state according to a new classification. 
(5) Anti-Acetylcholine Receptor Antibodies and Myasthenia Gravis

[\%]

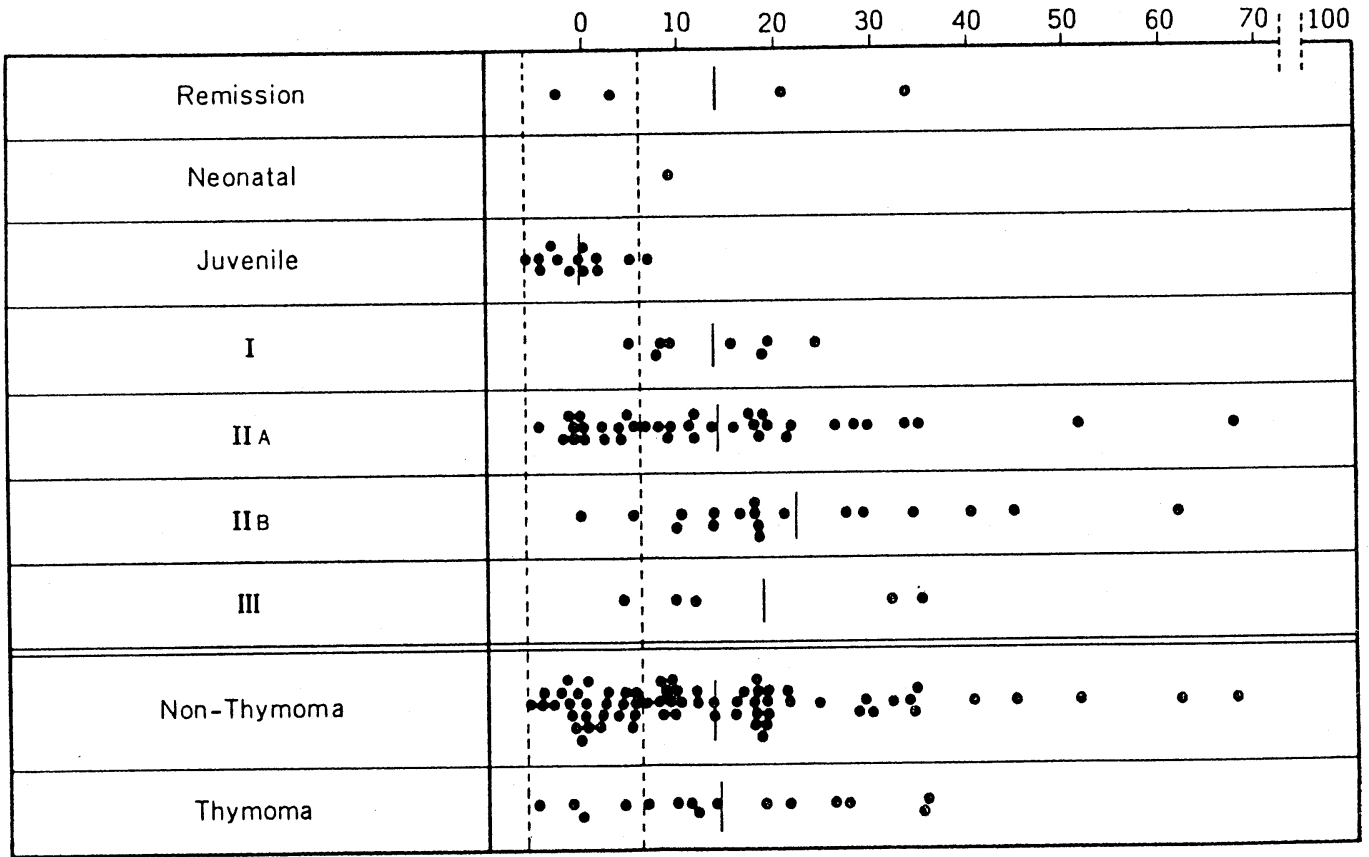

Fig. 2. Relationship between inhibitory activity by Concanavalin A Sepharose assay in myasthenic patients and their clinical state according to Ossrman's classification.

Ocular Non-Thymoma Group

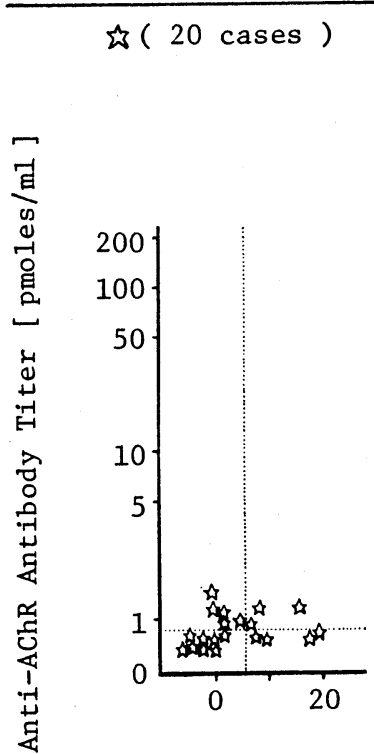

Generalized Non-Thymoma Group

( 45 cases )

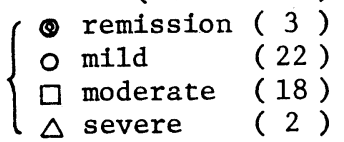

(3)

( 18 )

(2)
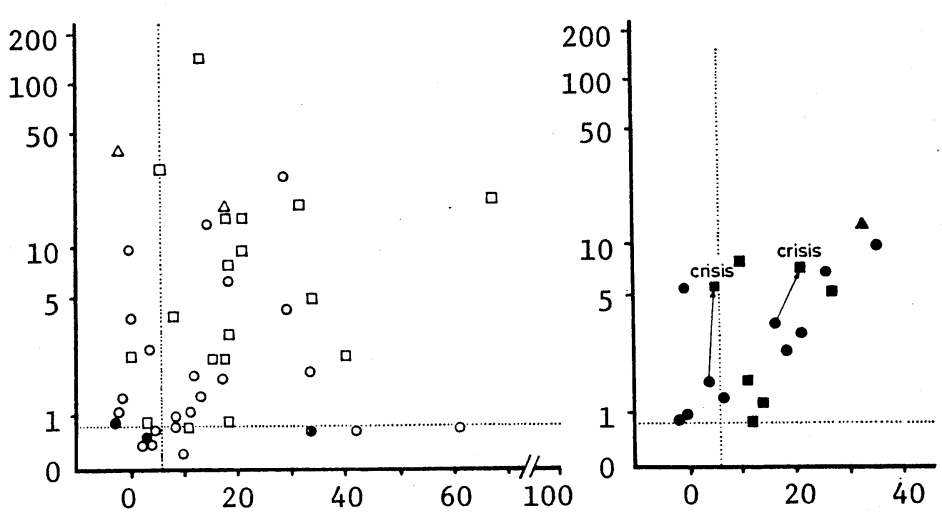

Inhibitory Activity [\%]
Thymoma Group

( 16 cases )

- remission ( 1 )

- mild

(9)

moderate (5)

(1)

Fig. 3. Relationship between anti-AChR antibody titer by immunoprecipitation method and inhibitory activity by Con A Sepharose method in the same sera of 81 myasthenics.

Jap J Med Vol 20, No 1 (Jan 1981) 
We obtained positive result in 53 out of $70(73 \%)$ myasthenics using Concanavalin A method, assuming that the antibody recognizes the neurotoxin binding site and/or sugar chain on AChR. There was more loose correlation of the inhibitory activity of the inhibitory antibody with the clinical state, comparing with the case in non-inhibitory antibody (Fig. 2).

Comparative and simultaneous assays in 81 myasthenic patients using the immunoprecipitation and concanavalin A method were carried out (Fig. 3). In 20 cases of ocular type the titers of both antibodies were low comparing with other myasthenic groups. In 16 cases of thymoma group there was a significant correlation at 5\% level between both antibody titers. On the contrary, in 45 cases of generalized nonthymoma group they were diverse and scattered without significant correlation each other. These data suggest that there may be different types of immune responses producing heterogeneous anti-AChR antibodies depending upon the types of myasthenia gravis and/or clinical conditions. This could elucidate part of the occasional discrepancies between the antibody titers and clinical symptoms.

The author measured the concentration of anti-AChR antibody in a paired serum and thymus obtained from 12 myasthenics and 3 control patients. All the myasthenic thymus extracts showed 1.4 to 16.8 times higher concentration of the antibody compared with that in the sera.

These data suggest the thymus of the myasthenic patient may be one of the site to produce anti-AChR antibody, although the estimated amount of anti-AChR antibodies in the thymus is not enough to explain the whole modulation process of the antibody at the neuromuscular junction in the generalized myasthenic patient.

\title{
(6) Lymphoreceptopathy in the Patients with Systemic Lupus Erythematosus
}

\author{
Shigeru ARIMORI, MD \\ The Fourth Department of Internal Medicine, School of Medicine, \\ Tokai University, Isehara-shi, Japan 259-11
}

\section{INTRODUCTION}

The anti-membrane antibody in the sera of the patients with systemic lupus erythematosus (SLE) was reported in 1972 without definite observation of receptor abnormali$\operatorname{ties}^{1)}$.

$\mathrm{T}$ lymphopenia ${ }^{2)}, \mathrm{T}_{\gamma}$ lymphopenia ${ }^{3)}$, decreased mitogenic activities against PWM and $\mathrm{PHA}^{2)}$, and reduced suppressor effect of $\mathrm{T}$ lymphocytes ${ }^{4)}$ were also determined previously.

The present paper entitled lymphore- ceptopathy in SLE has included further investigations obtained from the lymphocyte culture, membrane microviscosity, and freeze-fractured ultramicroscopy of SLE T lymphocytes in comparison with healthy $\mathrm{T}$ lymphocytes, been connected with the effect of SLE sera (IgG or/and IgM) in vitro on healthy $\mathrm{T}$ lymphocytes.

\section{MATERIALS AND METHODS}

The T lymphocytes were separated from 6 healthy subjects and 15 SLE patients through Conray-Ficoll gradient sedimenta- 